\title{
La institucionalización de los adultos dependientes. Características de la atención pública a nivel nacional y en Nuevo León.
}

\section{The institutionalization of dependant adults. Characteristics of the public attention at a national level and in Nuevo León.}

\author{
José Alejandro Meza Palmeros ${ }^{1}$ \\ Universidad Autónoma de Nuevo León (México) \\ Mónica Minerva Martínez Segura \\ Miembro del colectivo Re de Salud (México)
}

Fecha de recepción: 11 de julio de 2017

Fecha de aceptación: 27 de octubre de 2017

Disponible en línea: 28 de febrero de 2018

\section{Resumen}

El objetivo del presente trabajo es caracterizar la situación social de los adultos dependientes institucionalizados en México y Nuevo León, para lo cual se revisaron diversas fuentes oficiales de información: cifras correspondientes al Censo Nacional de Población y Vivienda; Dirección General de Información en Salud; Directorio Estadístico Nacional de Unidades Económicas y del Presupuesto de Egresos de la Federación de los años 2012 al 2017 y el gasto público en salud a través de la plataforma del Subsistema de Cuentas en Salud. Así mismo, se integró información

${ }^{1}$ E-mail: alefijomp@gmail.com

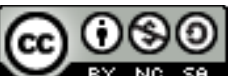

Revista del Centro de Investigación. Universidad La Salle por Dirección de Posgrado e Investigación. Universidad La $\underline{\text { Salle Ciudad de México se distribuye bajo una Licencia Creative Commons Atribución-NoComercial-CompartirIgual }}$ 
Palmeros Meza, J.; Martínez Segura, M.

solicitada vía Instituto Federal de Acceso a la Información a través de la plataforma de transparencia. A partir del análisis de la información recabada se señala un importante descuido de la atención a la dependencia tanto en México como en Nuevo León, dentro de un marco de reducción presupuestal que afecta particularmente a los adultos mayores cuya institucionalización y acogida ha sido derivada fundamentalmente al ámbito privado y a la caridad.

Palabras clave: Institucionalización; cifras estadísticas; adultos mayores; México; Nuevo León. 


\section{Abstract}

The objective of the present study is to distinguish the social situation of institutionalized dependant adults in Mexico and Nuevo León, for that purpose, various official sources of information were reviewed: corresponding figures to the National Population and Housing Census; General Directorate of Health Information; National Statistical Directory of Economic Units and Expenditure Budget of the Federation from the years 2012 to 2017 and the public spending on health through the platform of the Health Accounts subsystem.. Additionally, we integrated the requested information acquired from the Federal Institute of Access to Information through the transparency platform. Based on the analysis of the collected information, there is a significant neglect on the attention of the dependency in both Mexico and Nuevo León, within a framework of budget reduction that particularly affects older adults whose institutionalization and reception has been derived mainly from Private sphere and charity.

Keywords: Institutionalization, statistic figures, elders, México, Nuevo León. 


\section{Introducción}

La Organización Mundial de la Salud (2008) estima que el 13\% de la población en el mundo presenta alguna condición de dependencia, es decir, que lo inhabilita para realizar incluso las actividades más básicas para sobrevivir como comer, vestirse o asearse. La subsistencia de esta población depende, entonces, del cuidado de alguien más. Este 13\% podría doblarse en el año 2050 afectando fundamentalmente a personas de bajos ingresos.

Existen situaciones que provocan una dependencia de carácter permanente y los cuidados a partir de su aparición tendrán que ser brindados de por vida. Muchas son las enfermedades que pueden derivar en esta situación; algunas deteriorando el cuerpo hasta llegar a la inmovilidad, otras, deteriorando la mente hasta la interrupción total de la comunicación con el medio.

Fuera del ámbito corporal y funcional, dentro del cual se ha clasificado la dependencia de acuerdo a la capacidad para desarrollar ciertas actividades consideradas básicas, (ABVI); instrumentales (AIVD) o avanzadas (AAVD) necesarias para desenvolverse en la cotidianidad (Collin, 1988), existen diversas tipologías que han intentado incorporar la dimensión social de la dependencia. Por ejemplo, Fraser y Gordon (1994) ubican los siguientes aspectos: dependencia económica, dependencia socio-legal, dependencia política y dependencia moral y psicológica. Sin embargo, existen otras aplicaciones al concepto, las cuales se indica que resultan de mayor relevancia para el estudio de la dependencia en adultos, especialmente en adultos mayores, como lo define Walker (1982) quien habla de la dependencia en el ciclo de vida, la dependencia física y psicológica, dependencia económica y financiera, y la dependencia estructural. Gibson (1998), también propuso una tipología que incluye las dependencias políticas, legales, psicológicas y emocionales, asociadas principalmente a la edad, a la presencia de enfermedades y a la fragilidad.

No obstante, como sugiere Birtchnell (1998), cada una de estas clasificaciones de dependencia, refieren a distintos aspectos de un mismo fenómeno, por lo que sus manifestaciones suelen ser muy diversas en cada caso. Para fines de este trabajo, se intenta caracterizar la situación de dependencia a partir de una de las respuestas sociales más relevantes a saber: la acogida de esta población en instituciones especializadas denominadas comúnmente como asilos, como se detallará posteriormente. 
Indudablemente es en la vejez donde se presenta con más frecuencia una situación de dependencia. Estudios llevados a cabo en México en esta población (mayores de 65 años) (Barrantes et al, 2007), han encontrado que hasta el 47\% de los individuos estudiados presenta algún tipo de dependencia. De este porcentaje, el 24\% corresponde a una afectación en el desarrollo de las actividades básicas de la vida diaria, manifestándose en el 6\% una forma considerada severa. A su vez, el deterioro cognitivo explica una gran parte de este último tipo de dependencia (Gutiérrez y Arrieta, 2015).

La Clasificación Internacional de Enfermedades en su décima versión señala 100 tipos de enfermedades mentales. De éstas únicamente cuatro tipos corresponden al grupo de las demencias, reconocidas por ser las que explican la mayor parte de las causas del deterioro cognitivo severo en la población adulta. Estos cuatro tipos de demencias que se mencionan son: por Alzheimer, demencias de origen vascular, demencias no especificadas y demencias clasificadas en otra parte, que integra demencias provocadas por causas infecciosas, metabólicas y otras más, sumando en total 42 entidades clínicas (OMS, 1992).

Se calcula que la prevalencia en México de demencia es del 5\% de la población mayor a los 65 años (Mejía y Gutiérrez, 2011). Si consideramos que tan solo dentro de esta categoría médica se integran como ya mencionamos 42 enfermedades, sin contar las demás patologías que provocan un deterioro cognitivo, estamos hablando que existe una diversidad importante de causas que provocan una dependencia total.

Por diferentes motivos es cada vez más frecuente que los adultos dependientes terminen sus días en un lugar dispuesto para proveer cuidados en general denominado como casa de asistencia, estancia o asilo, que se incluye dentro de lo que Norton (2000) denomina «Cuidados a Largo Plazo» (CLP). Dichas estancias difieren de otro tipo de Cuidados a Largo Plazo como puede ser aquellos otorgados mediante una red informal como familiares y amigos, en que los cuidados son brindados, al menos en pretensión, mediante instituciones y agentes profesionales. Este fenómeno es una tendencia a nivel mundial y se desarrolla, sobre todo, en sociedades industrializadas (Carrière, 1993).

Como apunta Varul (2010), un elemento relevante para el caso de la institucionalización de adultos dependientes, consiste en la pérdida de valor y estima otorgados socialmente, debido a un deterioro irreversible en la autonomía y la consiguiente interrupción de sus funciones en la producción, principalmente en sociedades donde la participación en el sistema productivo 
constituye un elemento fundamental para el reconocimiento social. En este sentido Townsend (1981) señala, para el caso particular de adultos mayores, por citar un ejemplo, cuyas implicaciones en la dependencia son conocidos, que la proliferación de instituciones para el cuidado de este grupo, en los inicios del siglo XX, se encuentra estrechamente relacionada a la modificación del sistema social de producción, comenzando a considerarse la nula participación de un creciente sector de la población en el proceso productivo, un importante problema social.

De manera distinta, el cuidado brindado a adultos dependientes en algunas sociedades no industrializadas estudiadas, se realiza fundamentalmente a través de redes de apoyo de tipo religioso, lazos familiares y relaciones comunitarias solidarias, aunque de manera subordinada al grado de vinculación y afecto que se ha mantenido con la persona durante su vida, así como el grado de prestigio, veneración y reconocimiento con el que cuente (Reyes, 2012).

No obstante, podemos afirmar que para los distintos modelos, la posición social del sujeto, ya sea ésta sostenida a partir de los recursos económicos disponibles -consecuencia clara de su rol en el sistema productivo-, o a partir del afecto y prestigio, constituye un determinante central en la situación social y en las condiciones de vida del adulto dependiente. Esto aparece con mucha claridad en contextos con amplias desigualdades sociales, pobreza y un Estado para el que esta población no constituye una prioridad y que tiende a derivar el cuidado de estas personas a la familia y a la asistencia social, como es el caso de México.

A pesar de que no existe en México información oficial que describa la situación referente a la salud de los y las residentes de instituciones que brinden «Cuidados a Largo Plazo», y por lo tanto que permita conocer la prevalencia de dependencia en dichas casas de asistencia, es posible inferir, como un supuesto, que dicha prevalencia es alta, debido a que la dependencia de un individuo constituye uno de los motivos más importantes para que la familia opte por buscar cuidados y acogida profesionales (Meza y Escalera, 2017). Es por este motivo que al caracterizar a esta población, usuaria de CLP profesionales, se ha empleado información oficial respecto a las casas de asistencia, lo que evidentemente constituye una importante debilidad metodológica.

\section{Situación de dependencia de adultos mayores en México}

De acuerdo al INEGI (2014), existen 11.7 millones de adultos mayores en el país lo que representa al $0.7 \%$ de la población total. Aunque se considera que una gran parte de las casas que proveen 
cuidado permanente no se encuentran registradas, a partir de las que sí cuentan con un registro oficial se tiene conocimiento que en estas instituciones residen 22,611 personas, de las cuales el $61 \%$ son mujeres y poco más de la mitad (55\%) cuentan con una escolaridad máxima de primaria, de acuerdo al Censo de Alojamientos de Asistencia Social (INEGI, 2015).

Respecto a la salud de los adultos mayores, el INEGI (2014) estima que más del 20\% sufre algún trastorno mental o neurológico, siendo la demencia y la depresión los padecimientos de esta índole más frecuentes de acuerdo a los egresos hospitalarios reportados en el 2012 (Manrique et al, 2013).

En relación a la funcionalidad y discapacidad, algunos estudios (Gutiérrez et al, 2012) han calculado que de 8874 hombres y mujeres encuestados, que tienen 60 años o más y que representaron al 9.2\% de la población estimada del país en 2012, más de una cuarta parte de los adultos mayores, es decir, 26.9\%, presenta algún grado de discapacidad que compromete los aspectos básicos de la vida diaria (caminar, bañarse, acostarse o levantarse de la cama y vestirse), mientras que un $24.6 \%$ presentan dificultad para realizar al menos una de las cuatro actividades denominadas instrumentales (preparación de alimentos, compra de alimentos, administración de medicamentos y manejo de dinero). En ambos tipos de discapacidad la prevalencia incrementa a medida que aumenta la edad y es mayor en las mujeres. Esto ha sido registrado por Ham (1996), quien señala que las condiciones de salud y de incapacidad en México, tienen incidencias mayores a partir de los 75 años de edad, y además ocurren más temprano y son más significativas en la población femenina. Esto nos indica que existe una doble desprotección a este sector de la población, por ser mujeres y al ser mayores de 75 años. Esto indudablemente constituye un reto importante para el Estado, ya que la atención a la salud se torna insuficiente mientras más avanzan los procesos de envejecimiento, asociado, como ha sido señalado, a una situación de dependencia.

Un aspecto que ha cobrado bastante interés debido al papel que tiene para el desarrollo de discapacidad y dependencia en los adultos mayores es el daño sufrido a consecuencia de caídas. De acuerdo a la Encuesta Nacional de Salud y Nutrición (Gutiérrez et al, 2012) al menos 34\% de esta población sufrió una caída en los doce meses previos a la encuesta; siendo las mujeres afectadas con mayor frecuencia.

Situación de dependencia de Adultos Mayores en Nuevo León 
De acuerdo al Censo de Población y Vivienda (INEGI, 2010), en Nuevo León existen 407,278 adultos mayores lo cual representa el $10 \%$ de la población total para dicha entidad. De esta población se estima que 1,842 personas residen en alojamientos de asistencia social de la cuales el $66 \%$ son mujeres, un porcentaje superior a lo que se observa a nivel nacional. De esta población residente en casas de asistencia, el 53\% tienen una escolaridad inferior a la básica. Aunque, por supuesto, este dato no es suficiente para tener una mejor idea respecto a la posición social, si es posible plantear, al menos hipotéticamente, que la posición socioeconómica es un determinante importante de la institucionalización en Nuevo León.

En relación a la salud, de acuerdo a los egresos hospitalarios del año 2016 reportados por el sistema de salud (DGIS, 2017), los traumatismos constituyen la causa de hospitalización más frecuente, seguida de las enfermedades del corazón, hernias, enfermedades de la vesícula biliar y enfermedades cerebrovasculares como hemorragia o isquemia cerebral. Es decir, la mayor parte de causas de hospitalización que se presentan son potencialmente generadoras de dependencia severa.

\section{Características de las casas de asistencia en México y Nuevo León}

El Directorio Estadístico Nacional de Unidades Económicas (DENUE, 2017) refiere que en todo el país existen registrados 1,808 centros para el cuidado de adultos, de los cuales $51 \%$ son privados. De estos centros, de acuerdo al Censo de Alojamientos de Asistencia Social (INEGI, 2015) el 56\% $(1,020)$, brindan además de cuidado alojamiento permanente. Respecto a la figura jurídica de este $56 \%$ denominados casas hogar, únicamente el 5\% corresponden a instituciones públicas; el 95\% restante constituyen instituciones conformadas por la iniciativa privada de tipo religioso, asociaciones civiles, organismos de asistencia o instituciones de financiamiento privado.

La información relacionada con la infraestructura y los servicios ofertados se presentan en la tabla 1 .

Por otro lado, puede apreciarse que la mayoría de las casas hogar, tanto a nivel nacional como en Nuevo León registradas, tienen un espacio físico que permite ofertar de 5 a 20 habitaciones y de 10 a 20 camas. (Gráficas 1 y 2). 
Gráfica 1. Número de camas por casa hogar en México.

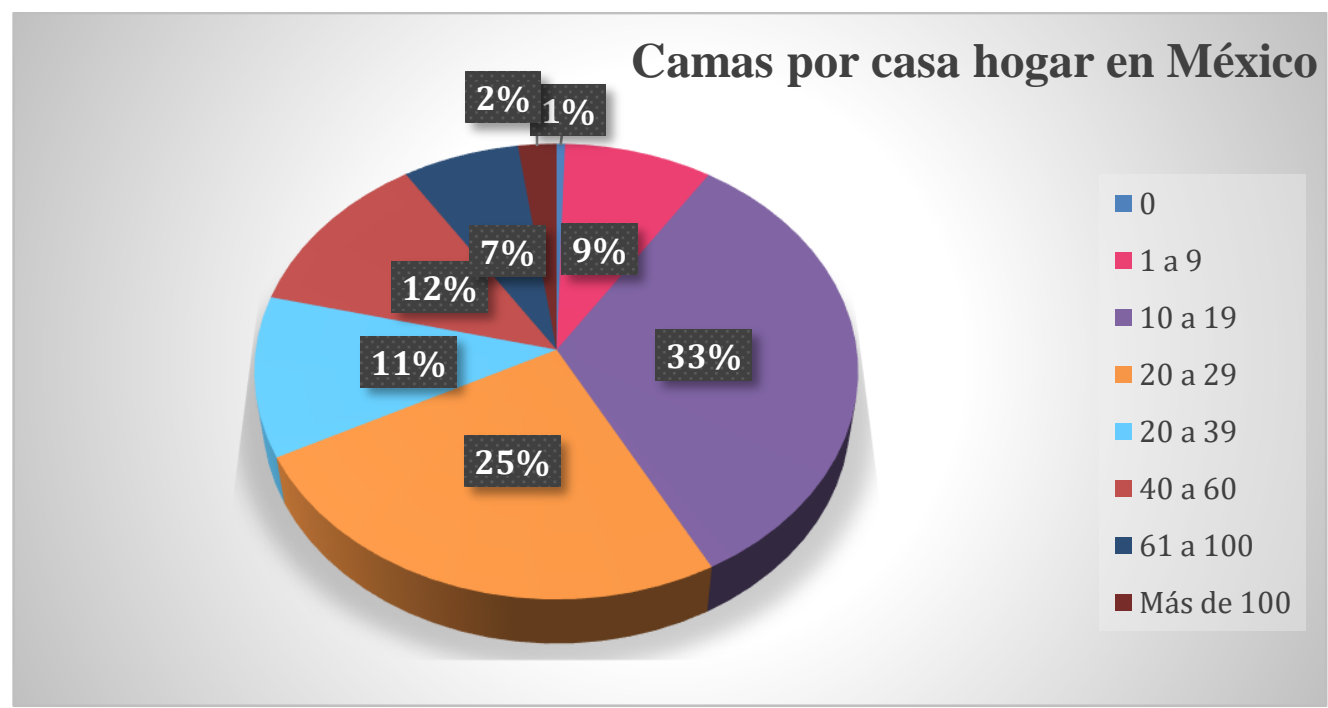

Fuente: elaboración propia a partir de INEGI. Tabulados del Censo de Alojamientos de Asistencia Social 2015 (Alojamiento), consultado el 8 de mayo, 2017 en: http://www.beta.inegi.org.mx/proyectos/encotras/caas/2015/

Gráfica 2: Número de camas por casa hogar en Nuevo León.

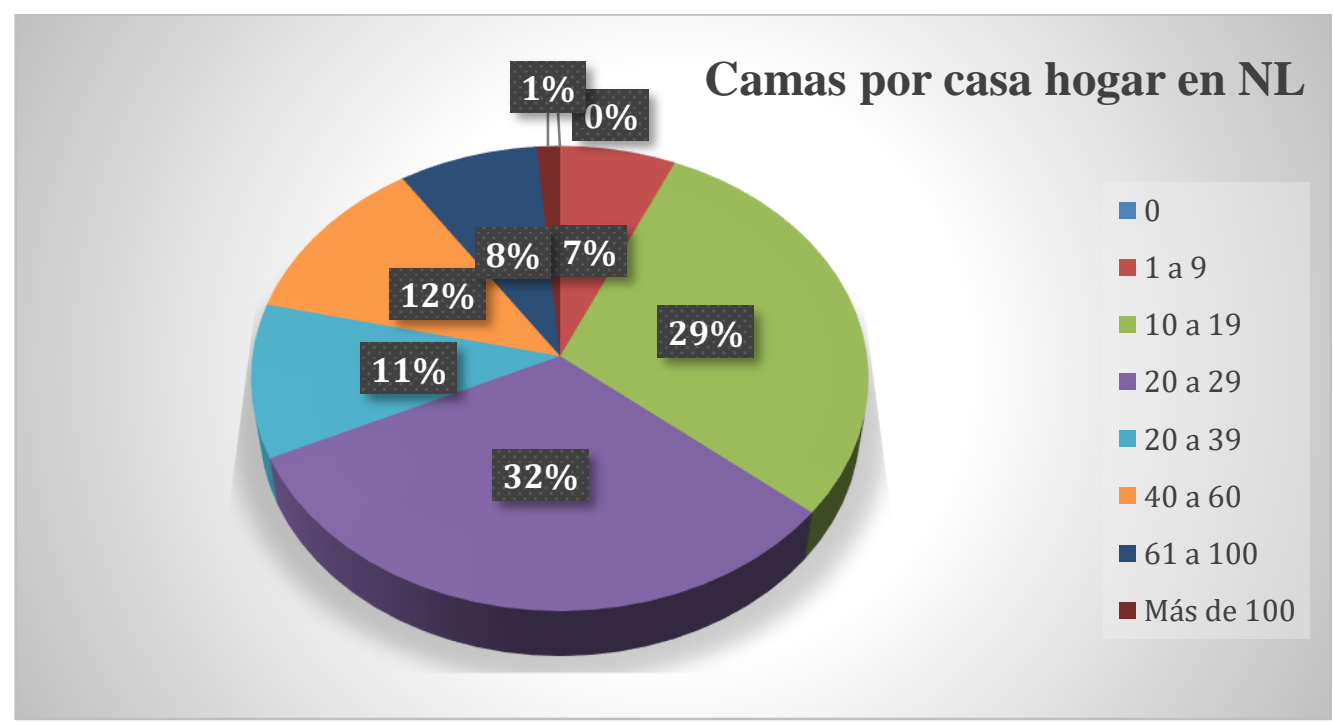

Fuente: elaboración propia a partir de INEGI. Tabulados del Censo de Alojamientos de Asistencia Social 2015 (Alojamiento), consultado el 8 de mayo, 2017 en: http://www.beta.inegi.org.mx/proyectos/encotras/caas/2015/ 
Respecto a la disponibilidad de instalaciones, el perfil de las casas entre lo observado a nivel nacional y Nuevo León es muy similar destacando en ambos ámbitos una insuficiencia de espacios para rehabilitación física.

En relación al tiempo de permanencia, la discrepancia que aparenta la información en cuanto a que las cifras parecen ser mayores que el número de casas hogar, se debe a que un adulto es registrado en varias ocasiones. La información indica una tendencia a la permanencia a largo plazo, pero también es posible inferir que existe bastante movilidad entre las diferentes residencias. (Gráficas 3 y 4).

Gráfica 3: Tiempo de permanencia de adultos en casas hogar en México.

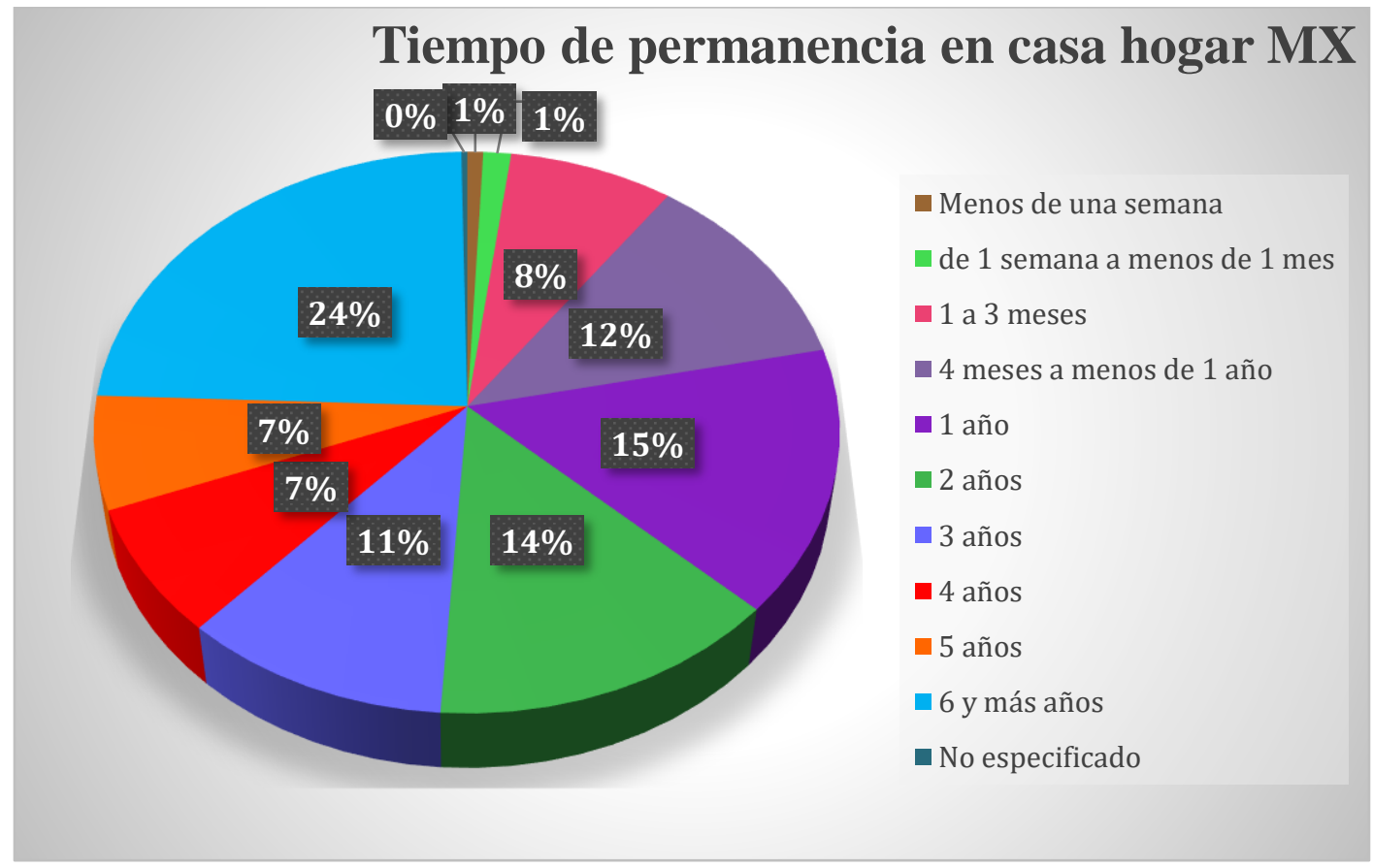

Fuente: elaboración propia a partir de INEGI. Tabulados del Censo de Alojamientos de Asistencia Social 2015 (Usuarios), consultado el 8 de mayo, 2017 en: http://www.beta.inegi.org.mx/proyectos/encotras/caas/2015/ 
Gráfica 4. Tiempo de permanencia de adultos en casa hogar en Nuevo León

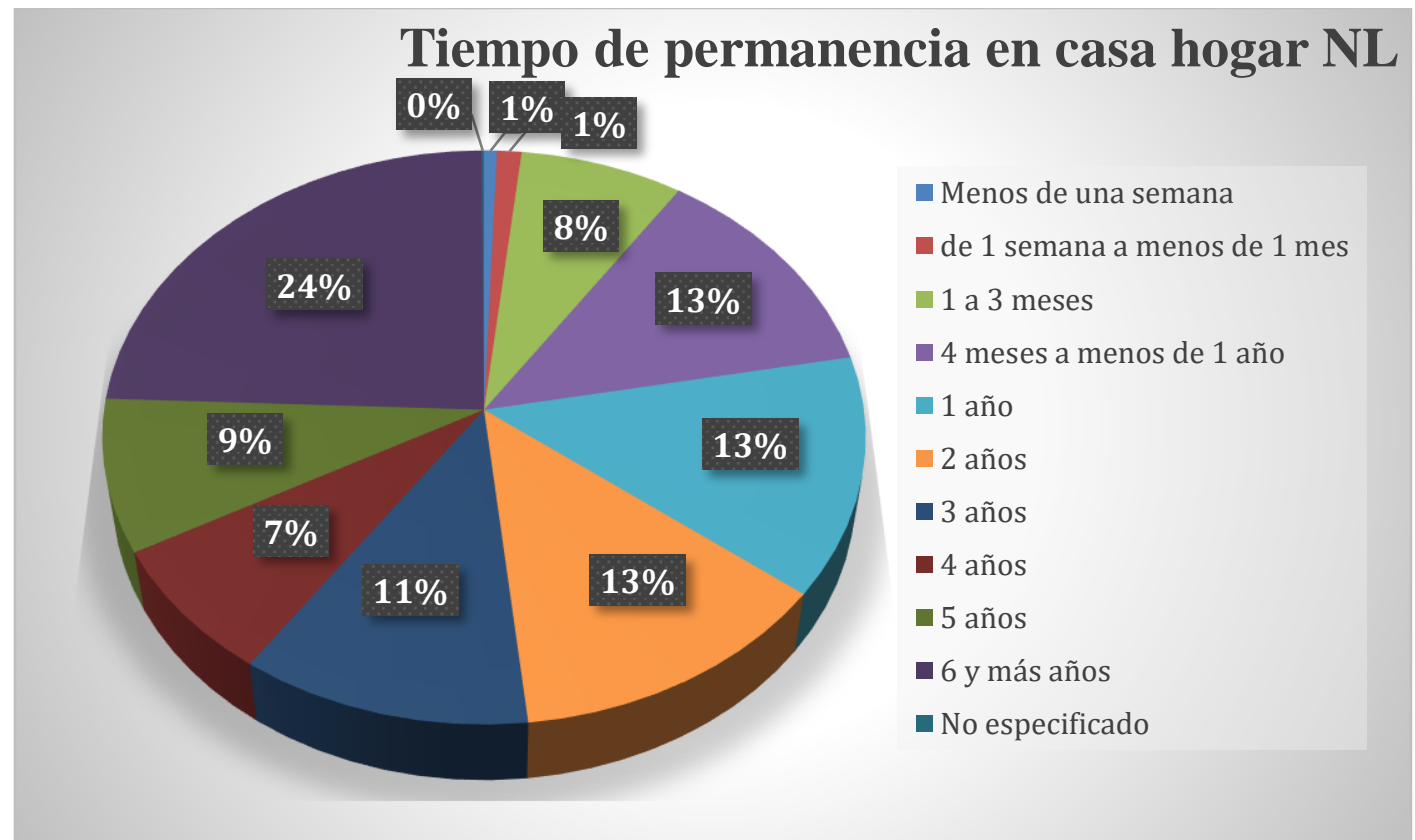

Fuente: elaboración propia a partir de INEGI. Tabulados del Censo de Alojamientos de Asistencia Social 2015 (Usuarios), consultado el 8 de mayo, $2017 \mathrm{en:} \mathrm{http://www.beta.inegi.org.mx/proyectos/encotras/caas/2015/}$

En cuanto a los servicios otorgados destaca que en ambos niveles de observación (nacional y estatal), el porcentaje más bajo lo presenta el servicio de psicología (Gráfica 5).

Gráfica 5. Disponibilidad de servicios en casas hogar en México y Nuevo León 


\section{Disponibilidad de servicios en casas hogar: asilos}

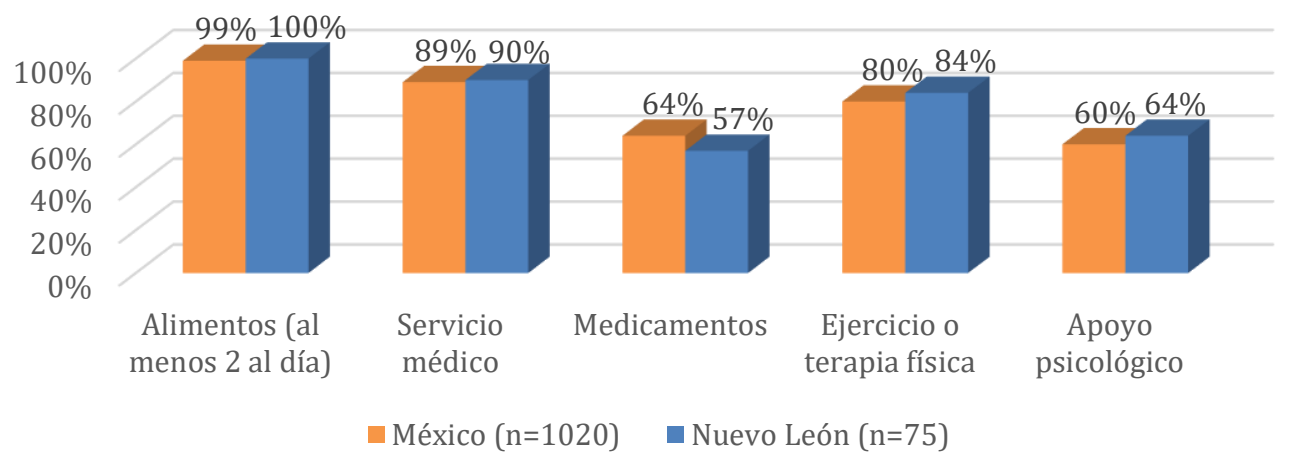

Fuente: elaboración propia a partir de INEGI. Tabulados del Censo de Alojamientos de Asistencia Social 2015 (Alojamiento), consultado el 8 de mayo, 2017 en: http://www.beta.inegi.org.mx/proyectos/encotras/caas/2015/

Esto definitivamente es un indicador que debe atenderse ya que, como hemos apuntado anteriormente, la depresión constituye una de las enfermedades más frecuentes en los adultos mayores lo que puede estar relacionado a un sentimiento de aislamiento y soledad que también es frecuente encontrar en este grupo de edad (Pelcastre et al, 2011).

En relación a las fuentes de financiamiento, los principales resultados se presentan en la tabla 2, en donde se destaca el elevado porcentaje que representan las cuotas a familiares o residentes como fuente de financiamiento, seguido por las donaciones de particulares y es notoria la poca participación que tiene el gobierno. Viendo estos resultados podemos afirmar que las casas hogar se financian fundamentalmente de manera privada, teniendo la caridad un papel secundario, pero igualmente importante (Gráfica 6).

Gráfica 6. Fuentes de financiamiento de casas hogar en México y Nuevo León 


\section{Fuentes de financiamiento de casas hogar: asilos*}

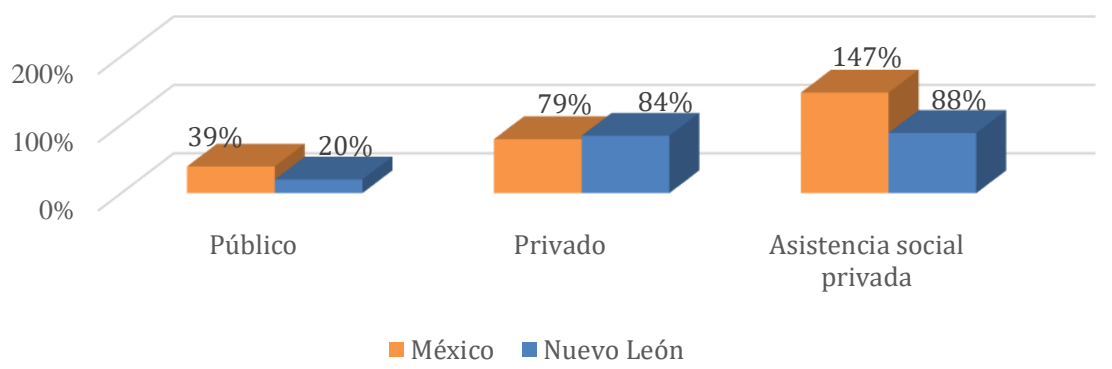

* Las casas pueden financiarse con más de una fuente

Fuente: elaboración propia a partir de INEGI. Tabulados del Censo de Alojamientos de Asistencia Social 2015 (Alojamiento), consultado el 8 de mayo, 2017 en: http://www.beta.inegi.org.mx/proyectos/encotras/caas/2015/

Programas e Instituciones responsables de los CLP profesionales y presupuesto dirigido a la atención de adultos dependientes

En el caso de México, la atención pública en torno al tema de adultos mayores se encuentra a cargo del DIF a través del programa de asistencia social E040 "Servicios de atención vulnerable", que opera únicamente en los estados de Morelos, Oaxaca y Ciudad de México, por lo que resulta ser, evidentemente, una respuesta insuficiente para atender a la población dependiente vulnerable económicamente. Otro programa similar es el S070 "Programa de Coinversión social" perteneciente al Instituto Nacional para el Desarrollo Social (INDESOL, 2016), que se encarga de financiar proyectos en beneficio de asilos y casas de asistencia.

Indiscutiblemente, uno de los aspectos que reflejan el nivel de prioridad que guarda determinado grupo poblacional dentro de la política social, es el presupuesto destinado para su atención. En relación a la política pública para la atención de la dependencia en México, podemos ubicarla a partir del seguimiento presupuestal de los programas dirigidos a los adultos mayores y las personas con discapacidad, debido a las implicaciones que tienen estas condiciones en la autonomía de los individuos.

Como se aprecia en la tabla 3, la atención a la dependencia en México puede localizarse a partir de tres fuentes de financiamiento: el presupuesto destinado a la salud, al desarrollo social y a algunas partidas presupuestales ubicadas dentro del mecanismo de protección a los Derechos Humanos. Como puede observarse, a partir del año 2015 existe una reducción global del presupuesto dirigido para atender la salud y para el desarrollo social de la población. Aún dentro de este recorte presupuestal, existe un notable incremento dirigido a la atención de las personas con 
discapacidad, a lo que se añaden etiquetamientos presupuestarios como el dirigido al Desarrollo integral de las personas con discapacidad ubicado dentro del rubro para el desarrollo social, que se incorpora a partir del año 2014 y del Programa de atención a personas con discapacidad, que se incorpora en el año 2007 como parte del rubro de la atención a la salud.

Sin embargo, también puede observarse la existencia de una marcada reducción del presupuesto particularmente para la atención de los adultos mayores, como el presupuesto para el Instituto Nacional de Geriatría, obligado a operar en el año 2016 y 2017 con un presupuesto correspondiente a una cuarta parte de lo asignado en el 2014; y del presupuesto para el Instituto Nacional de los Adultos Mayores, que sufrió en el año 2014 una reducción de prácticamente la mitad en comparación al año anterior 2013. Indudablemente esta reducción presupuestaria para atender las necesidades de este grupo poblacional de adultos mayores, se refleja en la poca participación que tiene el gobierno para financiar instancias de acogida sobre todo para aquella población más vulnerable en términos económicos.

\section{Gasto público en salud dirigido a los adultos mayores}

Como se aprecia en la tabla 4, el gasto público en salud dirigido para atender enfermedades crónico degenerativas muestra un importante recorte en los años 2013 y 2014 tanto en el ámbito nacional como en Nuevo león, siendo particularmente marcada esta reducción para la entidad. Es de destacar la interrupción del aporte económico otorgado en estos mismos años 2013 y 2014, por parte del Instituto de Seguridad y Servicios Sociales para los Trabajadores del Estado (ISSSTE), que había mantenido en los años previos una importante contribución en ambos ámbitos analizados, siendo como se aprecia, el principal mecanismo de financiamiento del programa en Nuevo León hasta el año 2013. Es notorio que como se observa, sin el aporte del ISSSTE, el gasto para la atención de la salud de los adultos mayores se reduce de manera muy importante en dicha entidad.

Para conocer mayores detalles respecto a esta importante reducción en el gasto, se realizó una solicitud a la Comisión de trasparencia y acceso a la información en Nuevo León por medio del sistema de Infomex, la cual fue dirigida al Congreso del Estado, pidiendo como información lo siguiente: 
La institucionalización de los adultos dependientes. Características de la atención pública a nivel nacional y en Nuevo León.

Presupuesto asignado para el estado de Nuevo León para el programa: "Elevar la calidad de vida del adulto mayor" en los años 2012, 2013 y 2014 de acuerdo a las siguientes fuentes: ramo 12, ramo 33 y Gobierno del Estado de Nuevo León.

Presupuesto público para operar programa de salud: función 7; subfunción 1 y 2; programa H37.

Dicha solicitud se realizó el día 28 de Mayo del presente año, teniendo como folio el número 00739517, sin embargo nunca se recibió respuesta ni notificación de prórroga.

El mismo día se realizó la misma solicitud, pero en página de la Plataforma Nacional de Transparencia, la cual generó el número de folio 0001200185817 y definió como plazo de entrega el día 26 de Junio del presente año. En esta ocasión la respuesta fue entregada el día 22 de Junio por medio de la plataforma nacional, informando lo siguiente:

En relación al presupuesto asignado para el estado de Nuevo León para el programa: "Elevar la calidad de vida del adulto mayor" en los años 2012, 2013 y 2014 del ramo 33:

Se muestra cuadro con los recursos asignados al Estado de Nuevo León en el Presupuesto de Egresos de la Federación (PEF) a través del FASSA del Ramo 33 en el programa H37 "atender el envejecimiento de los adultos y adultos mayores:

\begin{tabular}{|l|l|}
\hline PEF FASSA & IMPORTE \\
\hline 2012 & $7,398,696$ \\
\hline 2013 & $3,507,131$ \\
\hline 2014 & 58,046 \\
\hline TOTAL & $10,963,873$ \\
\hline
\end{tabular}

Fuente: Información brindada a través de la Plataforma Nacional de Transparencia, folio: 0001200185817.

Con respecto al Ramo 12, la Secretaría de Salud respondió que en los sistemas presupuestarios con los que cuenta la Dirección General, no se encuentra registrada la función 7 para los ejercicios 2012, 2013 y 2014 ni para el criterio de "Elevar la calidad de vida del adulto mayor" en el Estado de Nuevo León, y de igual modo, la Dirección General de Programación, Organización y Presupuesto se declaró incompetente al no conocer sobre la información solicitada en cuanto Ramo 12 y sobre el Gobierno del Estado de Nuevo León, por lo que sugirieron dirigir la solicitud a la Secretaría de Salud de esta entidad.

A pesar de no contar con la información respecto al gasto proveniente del Ramo 12 para Nuevo León, es de notar importantes discrepancias entre el presupuesto asignado vía FASSA (ramo 33) y el gasto en salud ejercido con el aporte de este mismo componente, reportado por el 
Subsistema de Cuentas en Salud. Al contrastar ambas fuentes, es posible observar una diferencia total entre lo presupuestado y el gasto reportado de casi ocho millones de pesos $(7,909,307)$ en los años 2012 y 2013. Estas discrepancias deberán ser investigadas necesariamente con una mayor profundidad.

\section{Conclusiones}

La atención a la dependencia debe constituir en México una prioridad para la política social, considerando que es de esperarse un importante incremento de esta condición en los próximos años, aparejada a los cambios demográficos que se viven tanto a nivel mundial como en México. No obstante, existe en este país y en Nuevo León, un importante descuido para atender a la población dependiente, lo cual se refleja particularmente en el sistema de institucionalización o acogida a esta población, en un contexto marcado por reducciones presupuestales para atender los problemas de salud y de desarrollo social, generando que este sistema de acogida sea financiado fundamentalmente de manera privada o a través de la caridad, lo que manifiesta un importante problema para la población desfavorecida económicamente y que no cuenta con un aporte suficiente para pagar la atención de sus allegados en situación de dependencia. Este contexto es marcadamente desfavorable para la población de adultos mayores quienes indiscutiblemente no constituyen una prioridad política en el país, lo cual se refleja en los importantes recortes presupuestales de programas dirigidos para atender a este grupo poblacional. 
La institucionalización de los adultos dependientes. Características de la atención pública a nivel nacional y en Nuevo León.

Anexos

Tabla 1. Infraestructura y servicios otorgados en las casas hogar a nivel Nacional y en Nuevo León.

\begin{tabular}{|c|c|c|}
\hline INFRAESTRUCTURA & NACIONAL $(n=1020)$ & NUEVO LEÓN (n=75) \\
\hline \multirow[t]{2}{*}{$\begin{array}{l}\text { Número de dormitorios } \\
\text { por casa hogar }\end{array}$} & $\begin{array}{l}1 \text { a } 2: 67(7 \%) \\
3 \text { a } 4: 140(14 \%) \\
5 \text { a 6: } 178(17 \%) \\
7 \text { a } 10: 232(23 \%) \\
11 \text { a } 20: 239(23 \%) \\
21 \text { a } 40: 105(10 \%) \\
\text { Más de } 40: 59(6 \%) \\
\text { Promedio de usuarios por } \\
\text { dormitorio: } 1.55\end{array}$ & $\begin{array}{l}1 \text { a } 2: 3(4 \%) \\
3 \text { a } 4: 7(9 \%) \\
5 \text { a } 6: 11(15 \%) \\
7 \text { a } 10: 23(30 \%) \\
11 \text { a } 20: 17(23 \%) \\
21 \text { a } 40: 10(13 \%) \\
\text { Más de } 40: 4(5 \%) \\
\text { Promedio de usuarios por } \\
\text { dormitorio: } 1.71\end{array}$ \\
\hline & $\begin{array}{l}0: 5(0.4 \%) \\
1 \text { a } 9: 89(8 \%) \\
10 \text { a } 19: 336(33 \%)\end{array}$ & $\begin{array}{l}0: 0 \\
1 \text { a } 9: 5(6 \%) \\
10 \text { a } 19: 22(29 \%)\end{array}$ \\
\hline & $\begin{array}{l}\text { ntro de Investigación de } \\
\text { Vol. } 12 \text {, No. } 48 \text {, julio-d }\end{array}$ & $\begin{array}{l}\text { ersidad La Salle } \\
\text {, 2017: } 95-120\end{array}$ \\
\hline
\end{tabular}




\begin{tabular}{|c|c|c|}
\hline $\begin{array}{l}\text { Número de camas por casa } \\
\text { hogar }\end{array}$ & $\begin{array}{l}20 \text { a } 29: 258(25 \%) \\
30 \text { a } 39: 116(11 \%) \\
40 \text { a 60: } 123(12 \%) \\
61 \text { a } 100: 70(6 \%) \\
\text { Más de } 100: 23(2 \%) \\
\text { Promedio de usuarios por } \\
\text { cama: } 0.75\end{array}$ & $\begin{array}{l}20 \text { a } 29: 24(32 \%) \\
30 \text { a } 39: 8(11 \%) \\
40 \text { a } 60: 9(12 \%) \\
61 \text { a } 100: 6(8 \%) \\
\text { Más de } 100: 1(1 \%) \\
\text { Promedio de usuarios por } \\
\text { cama: } 0.81\end{array}$ \\
\hline $\begin{array}{l}\text { Disponibilidad de espacios } \\
\text { e instalaciones }\end{array}$ & $\begin{array}{l}\text { Cocina: } 1015(99 \%) \\
\text { Comedor: } 1005(98 \%) \\
\text { Sala de convivencia grupal: } \\
658(65 \%) \\
\text { Consultorio médico o } \\
\text { enfermería: } 658(65 \%) \\
\text { Instalaciones para rehab. } \\
\text { física: } 331 \text { (32\%) } \\
\text { Jardines o áreas verdes: } 829 \\
(81 \%)\end{array}$ & $\begin{array}{l}\text { Cocina: } 75(100 \%) \\
\text { Comedor: } 73(99 \%) \\
\text { Sala de convivencia grupal: } \\
65(86 \%) \\
\text { Consultorio médico o } \\
\text { enfermería: } 52(69 \%) \\
\text { Instalaciones para rehab. } \\
\text { física: } 23(30 \%) \\
\text { Jardines o áreas verdes: } 58 \\
(77 \%)\end{array}$ \\
\hline $\begin{array}{l}\text { Tiempo de residencia en } \\
\text { casa hogar }\end{array}$ & $\begin{array}{l}\text { Menos de una } \\
\text { semana: } 170 \\
1 \text { semana a menos de } \\
1 \text { mes: } 298 \\
1 \text { a } 3 \text { meses: } 1768 \\
4 \text { meses a menos } \\
\text { de } 1 \text { año: } 2666 \\
1 \text { año: } 3458 \\
2 \text { años: } 3182 \\
3 \text { años: } 2412 \\
4 \text { años: } 1671 \\
5 \text { años: } 1472 \\
6 \text { y más años: } 5450 \\
\text { No especificado: } 64\end{array}$ & $\begin{array}{l}\text { Menos de una } \\
\text { semana: } 11 \\
1 \text { semana a menos de } \\
1 \text { mes: } 21 \\
1 \text { a } 3 \text { meses: } 140 \\
4 \text { meses a menos } \\
\text { de } 1 \text { año: } 233 \\
1 \text { año: } 248 \\
2 \text { años: } 237 \\
3 \text { años: } 208 \\
4 \text { años: } 137 \\
5 \text { años: } 158 \\
6 \text { y más años: } 447 \\
\text { No especificado: } 2\end{array}$ \\
\hline Disponibilidad de servicios & $\begin{array}{l}\text { Alimentos (al menos } 2 \text { al día): } \\
1016(99 \%) \\
\text { Servicio médico: } 910(89 \%) \\
\text { Medicamentos: } 685(64 \%) \\
\text { Ejercicio o terapia física: } \\
802(80 \%) \\
\text { Apoyo psicológico: } 612 \\
(60 \%)\end{array}$ & $\begin{array}{l}\text { Alimentos (al menos } 2 \text { al día): } \\
75(100 \%) \\
\text { Servicio médico: } 68(90 \%) \\
\text { Medicamentos: } 43(57 \%) \\
\text { Ejercicio o terapia física: } \\
63(84 \%) \\
\text { Apoyo psicológico: } 48(64 \%)\end{array}$ \\
\hline
\end{tabular}


La institucionalización de los adultos dependientes. Características de la atención pública a nivel nacional y en Nuevo León.

Fuente: elaboración propia a partir de INEGI. Tabulados del Censo de Alojamientos de Asistencia Social 2015 (Usuarios), consultado el 8 de mayo, 2017 en: http://www.beta.inegi.org.mx/proyectos/encotras/caas/2015/

Tabla 2. Fuentes de financiamiento de casas hogar a nivel nacional y en Nuevo León.

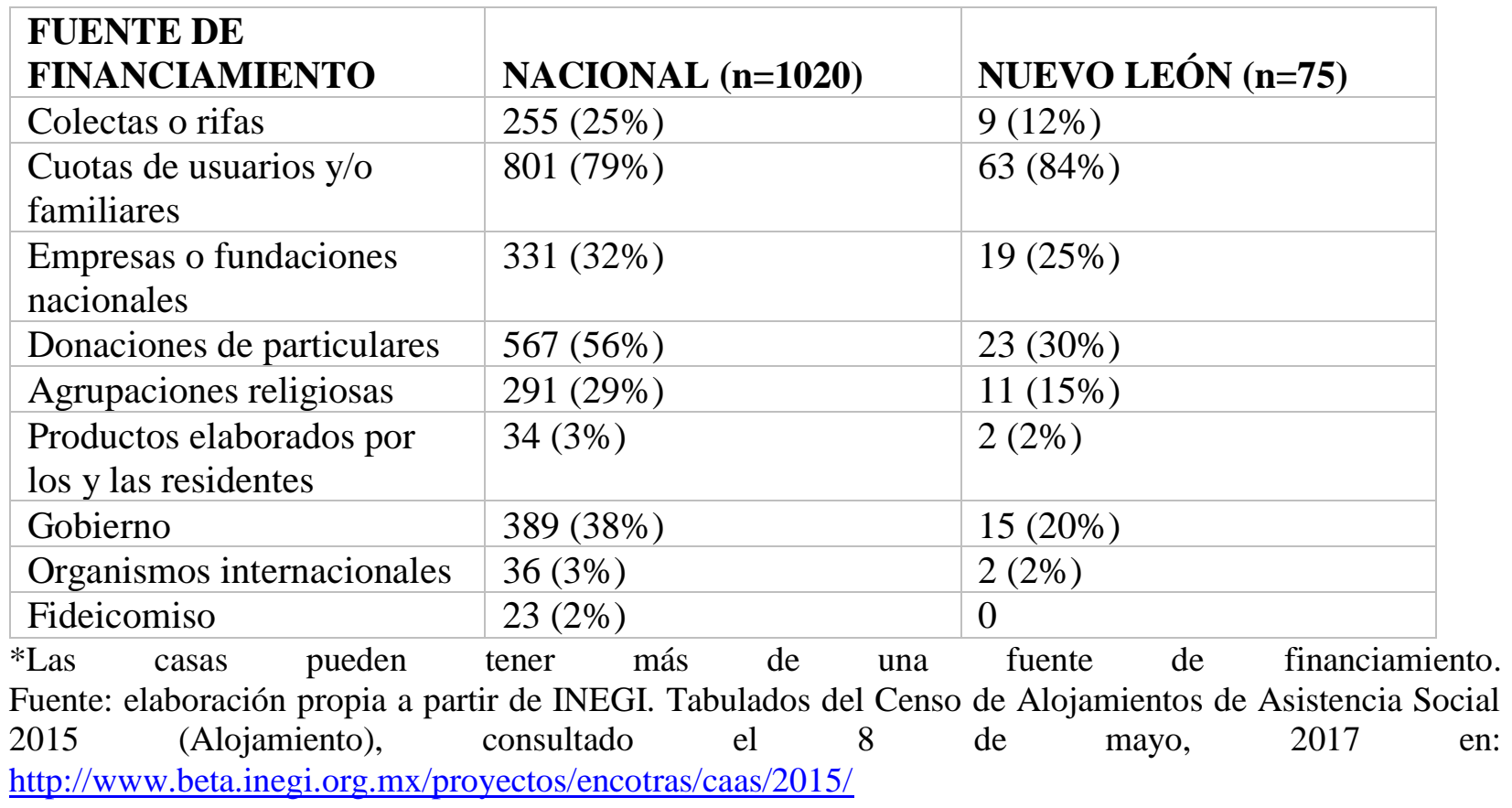

Tabla 3. Presupuesto asignado a salud, desarrollo social y atención a la dependencia 2012-2017

\begin{tabular}{|c|c|c|c|c|c|c|c|}
\hline \multirow{2}{*}{ Ramo } & \multirow{2}{*}{$\begin{array}{l}\text { Nombre/De } \\
\text { nominación }\end{array}$} & \multicolumn{6}{|c|}{ Presupuesto total asignado } \\
\hline & & 2012 & 2013 & 2014 & 2015 & 2016 & 2017 \\
\hline \multirow{4}{*}{ 12: Salud } & $\begin{array}{l}\text { Gasto total } \\
\text { en Salud }\end{array}$ & $113,479,679,217$ & $121,856,567,399$ & $130,264,761,743$ & $134,847,592,069$ & $132,216,881,794$ & $121,817,532,748$ \\
\hline & $\begin{array}{l}\text { Instituto } \\
\text { Nacional de } \\
\text { Geriatría }\end{array}$ & $38,965,897$ & $150,904,224$ & $222,650,665$ & $205,924,903$ & $54,134,714$ & $54,101,621$ \\
\hline & $\begin{array}{l}\text { Programa de } \\
\text { atención a } \\
\text { personas con } \\
\text { discapacidad }\end{array}$ & & & & & & $41,759,342$ \\
\hline & $\begin{array}{l}\text { Sistema } \\
\text { Nacional } \\
\text { para el } \\
\text { Desarrollo } \\
\text { Integral de la } \\
\text { Familia }\end{array}$ & $1,920,683,805$ & $2,123,166,064$ & $2,374,449,962$ & $2,516,135,619$ & $2,467,767,538$ & $2,273,267,537$ \\
\hline
\end{tabular}


Palmeros Meza, J.; Martínez Segura, M.

\begin{tabular}{|c|c|c|c|c|c|c|c|}
\hline & $\begin{array}{l}\text { Consejo } \\
\text { Nacional } \\
\text { para el } \\
\text { Desarrollo y } \\
\text { la Inclusión } \\
\text { de las } \\
\text { Personas con } \\
\text { Discapacidad }\end{array}$ & $3,610,831$ & $21,912,920$ & & & & \\
\hline \multirow{6}{*}{$\begin{array}{c}20: \\
\text { Desarroll } \\
\text { o Social }\end{array}$} & $\begin{array}{l}\text { Gasto total } \\
\text { en Desarrollo } \\
\text { Social }\end{array}$ & $84,859,857,194$ & $95,251,838,395$ & $111,211,236,932$ & $114,504,009,056$ & $109,371,909,018$ & $105,339,637,578$ \\
\hline & $\begin{array}{l}\text { Consejo } \\
\text { Nacional } \\
\text { para el } \\
\text { Desarrollo y } \\
\text { la Inclusión } \\
\text { de las } \\
\text { Personas con } \\
\text { Discapacidad } \\
\end{array}$ & & & $64,736,922$ & $78,160,879$ & $56,789,519$ & $57,594,726$ \\
\hline & $\begin{array}{l}\text { Instituto } \\
\text { Nacional de } \\
\text { las Personas } \\
\text { Adultas } \\
\text { Mayores } \\
\end{array}$ & $247,058,598$ & $609,949,932$ & $358,906,521$ & $370,314,281$ & $367,838,267$ & $373,423,483$ \\
\hline & $\begin{array}{l}\text { Delegación } \\
\text { SEDESOL } \\
\text { en Nuevo } \\
\text { León }\end{array}$ & $86,594,886$ & $102,102,401$ & $83,464,486$ & $83,565,138$ & $86,317,312$ & $67,717,056$ \\
\hline & $\begin{array}{l}\text { Servicios a } \\
\text { grupos con } \\
\text { necesidades } \\
\text { especiales }\end{array}$ & $223,367,138$ & $273,002,554$ & $301,155,703$ & $312,028,219$ & $321,701,088$ & $338,060,240$ \\
\hline & $\begin{array}{l}\text { Desarrollo } \\
\text { integral de } \\
\text { las personas } \\
\text { con } \\
\text { discapacidad }\end{array}$ & & & $64,334,620$ & $77,729,173$ & $56,397,690$ & $55,453,035$ \\
\hline \multirow{2}{*}{$\begin{array}{l}33: \\
\text { Aportaci } \\
\text { ones } \\
\text { Federales } \\
\text { para } \\
\text { Entidade } \\
\text { s } \\
\text { Federativ } \\
\text { as y } \\
\text { Municipi } \\
\text { os }\end{array}$} & $\begin{array}{l}\text { Gasto total } \\
\text { asignado a } \\
\text { Nuevo León }\end{array}$ & $15,355,549,469$ & $16,592,765,631$ & $17,673,271,045$ & $19,440,567,257$ & $19,846,015,879$ & $21,268,094,175$ \\
\hline & $\begin{array}{l}\text { Salud - } 19 \text { - } \\
\text { Nuevo León }\end{array}$ & $473,249,437$ & $489,069,777$ & $513,634,302$ & $737,495,726$ & $607,795,642$ & $659,130,077$ \\
\hline \multirow{2}{*}{$\begin{array}{c}\text { 35: } \\
\text { CNDH }\end{array}$} & $\begin{array}{l}\text { Gasto total } \\
\text { en CNDH }\end{array}$ & $1,280,276,629$ & $1,375,127,220$ & $1,416,381,046$ & $1,465,956,043$ & $1,546,934,935$ & $1,728,566,039$ \\
\hline & $\begin{array}{l}\text { Atender } \\
\text { asuntos de la }\end{array}$ & $4,211,907$ & $5,755,745$ & $5,812,629$ & $8,212,030$ & $8,638,924$ & $12,428,124$ \\
\hline
\end{tabular}

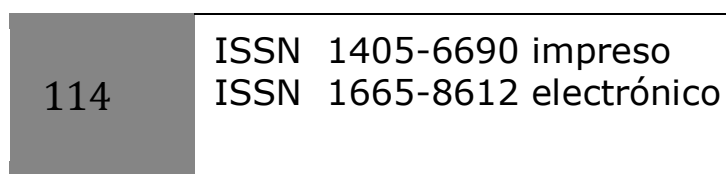




\begin{tabular}{|c|c|c|c|c|c|c|}
\hline $\begin{array}{l}\text { niñez, la } \\
\text { familia, } \\
\text { adolescentes } \\
\text { y personas } \\
\text { adultas } \\
\text { mayores. }\end{array}$ & & & & & & \\
\hline $\begin{array}{l}\text { Atender } \\
\text { asuntos } \\
\text { relativos a la } \\
\text { aplicación } \\
\text { del } \\
\text { Mecanismo } \\
\text { Nacional de } \\
\text { Promoción, } \\
\text { Protección y } \\
\text { Supervisión } \\
\text { de la } \\
\text { Convención } \\
\text { sobre los } \\
\text { derechos de } \\
\text { las Personas } \\
\text { con } \\
\text { Discapacidad }\end{array}$ & $4,171,092$ & $4,512,313$ & $7,140,900$ & $8,460,391$ & $9,806,955$ & $113,286,295$ \\
\hline
\end{tabular}

Fuente: elaboración propia a partir de la Secretaría de Hacienda y Crédito Público. (2017) Presupuesto de Egresos de la Federación 2012-2017 Consultado el 7 de abril de 2017 en http://www.pef.hacienda.gob.mx/

Tabla 4. Gasto público en salud a nivel nacional y en Nuevo León para operar el programa “elevar la calidad del adulto mayor” 2010-2014.

\begin{tabular}{|l|l|l|l|}
\hline AÑO & FUENTE & NACIONAL & NUEVO LEÓN \\
\hline \multirow{5}{*}{2010} & $\begin{array}{l}\text { FONDO DE APORTACIONES PARA LOS } \\
\text { SERVICIOS DE SALUD (RAMO 33) }\end{array}$ & 647127.29 & 1467.52 \\
\cline { 2 - 4 } & GASTO ESTATAL & 316085.91 & 18.49 \\
\cline { 2 - 4 } & $\begin{array}{l}\text { Instituto de Seguridad y Servicios Sociales de los } \\
\text { Trabajadores del Estado (ISSSTE) }\end{array}$ & 3866548.3 & 116910.00 \\
\cline { 2 - 4 } & $\begin{array}{l}\text { Instituciones de Seguridad Social de las Entidades } \\
\text { Federativas (ISSES) }\end{array}$ & 0 & 0 \\
\cline { 2 - 4 } & TOTAL & $\mathbf{4 , 8 2 9 7 6 1 . 5}$ & $\mathbf{1 1 8 , 3 9 6 . 0 1}$ \\
\hline \multirow{5}{*}{2011} & $\begin{array}{l}\text { FONDO DE APORTACIONES PARA LOS } \\
\text { SERVICIOS DE SALUD (RAMO 33) }\end{array}$ & 609334.57 & 0 \\
\cline { 2 - 4 } & GASTO ESTATAL & 203301.75 & 150.65 \\
\cline { 2 - 4 } & $\begin{array}{l}\text { Instituto de Seguridad y Servicios Sociales de los } \\
\text { Trabajadores del Estado (ISSSTE) }\end{array}$ & $\mathbf{2 8 7 5 4 1 0 . 4 2}$ & $\mathbf{7 2 6 3 2 . 8 2}$ \\
\cline { 2 - 4 } & $\begin{array}{l}\text { Instituciones de Seguridad Social de las Entidades } \\
\text { Federativas (ISSES) }\end{array}$ & 0 & 0 \\
\cline { 2 - 4 } & TOTAL & $\mathbf{3 , 6 8 8 0 4 6 . 7 4}$ & $\mathbf{7 2 , 7 8 3 . 4 7}$ \\
\hline
\end{tabular}


Palmeros Meza, J.; Martínez Segura, M.

\begin{tabular}{|c|c|c|c|}
\hline \multirow{5}{*}{2012} & $\begin{array}{l}\text { FONDO DE APORTACIONES PARA LOS } \\
\text { SERVICIOS DE SALUD (RAMO 33) }\end{array}$ & 986389.78 & 305.94 \\
\hline & GASTO ESTATAL & 161650.64 & 42.09 \\
\hline & $\begin{array}{l}\text { Instituto de Seguridad y Servicios Sociales de los } \\
\text { Trabajadores del Estado (ISSSTE) }\end{array}$ & 4126368.24 & 111115.21 \\
\hline & $\begin{array}{l}\text { Instituciones de Seguridad Social de las Entidades } \\
\text { Federativas (ISSES) }\end{array}$ & 65843.2 & 0 \\
\hline & TOTAL & $5,340251.86$ & $111,463.24$ \\
\hline \multirow{5}{*}{2013} & $\begin{array}{l}\text { FONDO DE APORTACIONES PARA LOS } \\
\text { SERVICIOS DE SALUD (RAMO 33) }\end{array}$ & 333971.45 & 2690.58 \\
\hline & GASTO ESTATAL & 67894 & 50.48 \\
\hline & $\begin{array}{l}\text { Instituto de Seguridad y Servicios Sociales de los } \\
\text { Trabajadores del Estado (ISSSTE) }\end{array}$ & 0 & 0 \\
\hline & $\begin{array}{l}\text { Instituciones de Seguridad Social de las Entidades } \\
\text { Federativas (ISSES) }\end{array}$ & 84257.9 & 0 \\
\hline & TOTAL & $486,123.35$ & 2741.06 \\
\hline \multirow{5}{*}{2014} & $\begin{array}{l}\text { FONDO DE APORTACIONES PARA LOS } \\
\text { SERVICIOS DE SALUD (RAMO 33) }\end{array}$ & 270784.27 & 82.56 \\
\hline & GASTO ESTATAL & 101291.07 & 11.88 \\
\hline & $\begin{array}{l}\text { Instituto de Seguridad y Servicios Sociales de los } \\
\text { Trabajadores del Estado (ISSSTE) }\end{array}$ & 0 & 0 \\
\hline & $\begin{array}{l}\text { Instituciones de Seguridad Social de las Entidades } \\
\text { Federativas (ISSES) }\end{array}$ & 103596 & 0 \\
\hline & TOTAL & $475,671.34$ & 94.44 \\
\hline
\end{tabular}

*Denominador en miles de pesos

Fuente: Dirección General de Información en Salud. (2015) Subsistema de Cuentas en Salud, México. http://www.dgis.salud.gob.mx/contenidos/sinais/s_sicuentas.html. Revisado el 10-03-2017 
La institucionalización de los adultos dependientes. Características de la atención pública a nivel nacional y en Nuevo León.

\section{Referencias}

Barrantes, M., García, E., Gutiérrez, L. M., \& Miguel, A. (2007). Functional dependence and chronic disease in older Mexicans. Salud Pública de México, 49, 459-466.

Birtchnell, J. (1988). Defining dependence. Psychology and Psychotherapy: Theory, Research and Practice, 61(2), pp. 111-123.

Carrière, Y., \& Légaré, J. (1993). Vieillissement démographique et institutionnalisation des personnes âgées: des projections nuancées pour le Canada. Cahiers québécois de démographie, 22(1), pp. 63-92. doi : http://dx.doi.org/10.7202/010135ar

Collin, C., Wade, D. T., Davies, S., \& Horne, V. (1988). The Barthel ADL Index: a reliability study. International disability studies, 10(2), 61-63. doi: http://dx.doi.org/10.3109/09638288809164103

Dirección General de Información en Salud. (2015) Subsistema de Cuentas en Salud, México. http://www.dgis.salud.gob.mx/contenidos/sinais/s_sicuentas.html Revisado el 10-03-2017

Fraser, N. \& Gordon, L. 1994. A genealogy of dependency: tracing a keyword of the US welfare state. Signs, 19, 2, pp. 309-34. doi: http://dx.doi.org/10.1086/494886 
Gibson, D. 1998. Aged Care: Old Policies, New Solutions. Cambridge University Press, Melbourne.

Gignac, M., \& Cott, C. (1998). A conceptual model of independence and dependence for adults with chronic physical illness and disability. Social science \& medicine, 47(6), 739-753. doi: http://dx.doi.org/10.1016/S0277-9536(98)00149-X

Gutiérrez, J., Rivera, J., Shamah, T., Villalpando, S., Franco, A., \& Cuevas L. (2012) Encuesta Nacional de Salud y Nutrición. Ensanut 2012 Resultados Nacionales. Cuernavaca, México: Instituto Nacional de Salud Pública.

Gutierrez, L., \& Arrieta, I. (2015). Dementia in Mexico: The need for a national Alzheimer's plan. Gac Med Mex, 151, pp. 667-673.

Ham, R. (1996). El envejecimiento: una nueva dimensión de la salud en México. Salud pública de México, 38(6).

Instituto Nacional de Estadística y Geografía. (2014). Estadísticas a propósito del día internacional de las personas de edad (1 Octubre) Datos nacionales. Aguascalientes.

Instituto Nacional de Estadística y Geografía. (2015) Directorio Estadístico Nacional de Unidades Económicas, Consultado el 16 de mayo, 2017 en: http://www.beta.inegi.org.mx/app/mapa/denue/

Instituto Nacional de Estadística y Geografía. (2015). Tabulados del Censo de Alojamientos de Asistencia Social 2015 (Usuarios), consultado el 8 de mayo, 2017 en: http://www.beta.inegi.org.mx/proyectos/encotras/caas/2015/

Manrique, B., Salinas, A., Moreno, K. M., Acosta, I., Sosa, A., Gutiérrez, L., \& Téllez, M. (2013). Condiciones de salud y estado funcional de los adultos mayores en México. Salud Pública de México, 55, pp. S323-S331.

Mejía-Arango, S., \& Gutiérrez, L. M. (2011). Prevalence and incidence rates of dementia and cognitive impairment no dementia in the Mexican population: data from the Mexican Health and Aging Study. Journal of aging and health, 23(7), pp. 1050-1074. doi: http://dx.doi.org/10.1177/0898264311421199

Meza, A, Escalera, L. (2017). Cuidar al otro: la carrera moral del adulto dependiente. México: editorial universitaria, UANL.

Norton, E. C. (2000). Long-term care. Handbook of health economics, 1, pp. 955-994.

Olivier, G. (2004). También pasan los años por los Dioses. Niñez, juventud y vejez en la cosmovisión mesoamericana. En Virginia Guedea (coord..), El historiador frente a la 
La institucionalización de los adultos dependientes. Características de la atención pública a nivel nacional y en Nuevo León.

historia: el tiempo en Mesoamérica. (pp. 149-180). México: Instituto de Investigaciones Históricas, UNAM.

Organización Mundial de la Salud. (1992). Clasificación Estadística Internacional de Enfermedades y Problemas Relacionados con la Salud. Revisado el 3 de marzo de 2017 en http://ais.paho.org/classifications/Chapters/pdf/Volume2.pdf

Organización Mundial de la Salud. (2008). Informe sobre la salud en el mundo 2008. La atención primaria de salud: Más necesaria que nunca. Revisado el 3 de marzo de 2017, de OMS Sitio web: http://www.who.int/whr/2008/es/

Parens, H. \& Saul, L. (1971). Dependence in Man. New York: International Universities Press.

Pelcastre, B., Treviño, S., González, T., \& Márquez, M. (2011). Apoyo social y condiciones de vida de adultos mayores que viven en la pobreza urbana en México. Cadernos de Saúde Pública, 27(3), pp. 460-470. doi: http://dx.doi.org/10.1590/S0102-311X2011000300007

Reyes, L. (2012). Etnogerontología social: la vejez en contextos indígenas. Revista del Centro de Investigación. Universidad La Salle, 10 (28). doi: http://dx.doi.org/10.26457/recein.v10i38.90

Secretaría de Hacienda y Crédito Público. (2017) Presupuesto de Egresos de la Federación 20122017. Consultado el 7 de abril de 2017 en http://www.pef.hacienda.gob.mx/

Secretaría de Salud. (2017). Dirección General de Información en Salud. Cubos Dinámicos: Egresos hospitalarios 2016 SAEH, Plataforma preliminar. Revisado el 10 de marzo de 2017 http://www.dgis.salud.gob.mx/contenidos/basesdedatos/bdc_egresoshosp_gobmx.html

Sistema Nacional para el Desarrollo Integral de la Familia (2016) E040 Servicios de Asistencia Social Integral-Atención Integral a las y los Adultos Mayores Sujetos de Asistencia Social. Sistema Nacional DIF. Publicado el 17 de Octubre de 2016 en: https://www.gob.mx/difnacional/acciones-y-programas/e040-servicios-de-asistenciasocial-integral-atencion-integral-a-las-y-los-adultos-mayores-sujetos-de-asistencia-social

Townsend, P. (1981). The structured dependency of the elderly: a creation of social policy in the twentieth century. Ageing and society, 1(01), pp. 5-28. doi: http://dx.doi.org/10.1017/S0144686X81000020

Varul, M. (2010). Talcott Parsons, the sick role and chronic illness. Body \& Society, 16(2), pp. 72-94. doi: http://dx.doi.org/10.1177/1357034X10364766

Walker, A. 1982. Dependency and old age. Social Policy and Administration, 16, pp. 115-35. doi: http://dx.doi.org/10.1111/j.1467-9515.1982.tb00164.x 
Palmeros Meza, J.; Martínez Segura, M. 